\title{
Urinary volatile organic compounds as potential biomarkers for renal cell carcinoma
}

\author{
DONGCHUN WANG, CHANGSONG WANG, XIN PI, LEI GUO, YUE WANG, \\ MINGJUAN LI, YUE FENG, ZIWEI LIN, WEI HOU and ENYOU LI
}

\author{
Department of Anesthesiology, The First Affiliated Hospital of Harbin Medical University, \\ Harbin, Heilongjiang 150001, P.R. China
}

Received February 1, 2016; Accepted April 25, 2016

DOI: $10.3892 /$ br.2016.686

\begin{abstract}
Currently, there is no adequate, sensitive, reproducible, specific and noninvasive biomarker that can reliably be used to detect renal cell carcinoma (RCC). Previous studies have elucidated the urinary non-volatile metabolic profile of RCC. However, whether urinary volatile organic compound (VOC) profiles are able to identify $\mathrm{RCC}$ remains to be elucidated. In the present study, urine was collected from 22 patients with RCC and 25 healthy subjects. Principal component analysis and orthogonal partial least square discriminant analysis were used to compare the data of patients and healthy subjects, and preoperative and postoperative patients undergoing radical nephrectomy. In total, 11 VOC biomarkers were elevated in the RCC patients compared to the healthy subjects, which were phenol; decanal; 1,6-dioxacyclododecane-7,12-dione; 1-brom o-1-(3-methyl-1-pentenylidene)-2,2,3,3-tetramethyl-cyclopropane; nonanal; 3-ethyl-3-methylheptane; isolongifolene-5-ol; 2,5-cyclohexadiene-1,4-dione, 2,6-bis(1,1-dimethylethyl); tetradecane; aniline; and 2,6,10,14-tetramethyl-pentadecane. Three biomarkers were decreased in RCC patients: styrene, 4-heptanone and dimethylsilanediol. In preoperative patients, 2-ethyl-1-hexanol and cyclohexanone were elevated, while 6-t-butyl-2,2,9,9-tetramethyl-3,5-decadien-7-yne were decreased when compared to postoperative patients. Compared
\end{abstract}

Correspondence to: Dr Enyou Li or Dr Changsong Wang, Department of Anesthesiology, The First Affiliated Hospital of Harbin Medical University, 23 Youzheng Street, Harbin, Heilongjiang 150001, P.R. China

E-mail: enyouli@aliyun.com

E-mail: changsongwang@aliyun.com

Abbreviations: RCC, renal cell carcinoma; VOC, volatile organic compound; GC/MS, gas chromatography/mass spectrometry; SPME, solid-phase microextraction; PCA, principal component analysis; PLSDA, partial least-squares discriminant analysis; VIP, variable importance in the projection

Key words: renal cell carcinoma, volatile organic compound, biomarker, diagnosis, urine with the healthy subjects, RCC has a unique VOC profile, suggesting that VOC profiles may be a useful diagnostic assay for RCC.

\section{Introduction}

The worldwide incidence of renal cell carcinoma (RCC), a predominantly male (2:1 ratio) disease, is $\sim 271,000$ new cases and 116,000 fatalities per year (1). There are several known risk factors for RCC, such as obesity, hypertension and decreased fruit and vegetable consumption. A subset of patients with this cancer has local or systemic symptoms, including flank pain, hematuria, a palpable abdominal mass, fever and wasting syndromes (2). However, in recent years, approximately half of the RCC patients have had no clinical symptoms; the disease was identified by abdominal imaging during a health check-up. The traditional method for clinical diagnosis is abdominal imaging. Computed tomography can be used to stage the primary tumor and determine the lymphadenopathy and metastasis (3), and Doppler sonography can better detect the tumor extension in equivocal cases (4). However, despite its sensitivity, imaging is relatively non-specific; therefore, the disease must be confirmed using renal biopsy. Although urine cytology, a reproducible and noninvasive approach, may be valuable for differentiating urothelial cell carcinoma from RCC (5), it lacks sensitivity. Currently, there is no adequate sensitive, reproducible, specific and noninvasive biomarker that can reliably be used to detect RCC.

In recent years, an increasing number of researchers have focused on volatile organic compound (VOC) profiling, a novel and robust procedure, because it is convenient and inexpensive. Altomare et al (6) confirmed that the pattern of breath VOCs in patients with colorectal cancer was different from healthy controls. Ikeda et al (7) proposed serum VOC profiling as a novel diagnostic approach for gastrointestinal cancer. Wu et al (8) demonstrated that hepatocellular carcinoma patients had a unique urinary VOC profile. Filipiak et al (9) demonstrated that lung cancer-derived cells exhibited specific volatile compounds. These studies indicate that different cancers may produce different VOCs profiles. Numerous metabolic profiling studies have focused on the urinary levels of nucleosides as biomarkers of cancers, including leukemia (10), breast cancer (11), colorectal cancer (12) and 
hepatocellular carcinoma (13), which are detected independent of patient age or gender. Metabolomics can also be used to elucidate biomarkers. Ganti and Weiss (14) obtained the urinary non-volatile metabolic profiles of RCC. Notably, Jobu et al (15) proposed that urinary VOC metabolites in human bladder cancer were significantly more sensitive compared to urine cytology. However, whether VOC profiling can be used to identify RCC remains to be elucidated.

In the current study, gas chromatography/mass spectrometry (GC/MS) and multivariate data analysis were used to compare the VOCs in urine samples from RCC patients (preoperative and postoperative) and healthy subjects to identify potential volatile biomarkers of RCC in VOCs.

\section{Materials and methods}

Patients. All the experimental protocols were approved by the Ethics Committee of the First Affiliated Hospital of Harbin Medical University (Harbin, Heilongjiang, China; no. 201314), and the informed consent was obtained from the parents of the patients prior to study enrollment. A total of 22 patients who were admitted to the Department of Urology (The First Affiliated Hospital of Harbin Medical University) between May 2011 and October 2012 were selected for the study (the cancer group). These 22 patients were diagnosed with RCC. In addition to the cancer group, 25 healthy volunteers were also included in the study.

Of the 22 patients with RCC, 15 were males and 7 were females. The mean age of the patients was $57.65 \pm 10.92$ years. Among them, 18 patients (12 males and 6 females) were involved in the preoperative and postoperative collection. The mean age was $58.22 \pm 11.29$ years. The normal control group of 25 included 12 males and 8 females. The mean age of individuals in the normal control group was $57.92 \pm 10.81$ years.

Urine sample collection. Urine samples $(2 \mathrm{ml})$ were immediately transferred to evacuated $20 \mathrm{ml}$ heparinized glass vials (Supelco, Inc., Bellefonte, CA, USA) before surgery and 1 day after surgery. All the samples were analyzed within $2 \mathrm{~h}$ of collection.

Solid-phase microextraction (SPME). For SPME, 75- $\mu \mathrm{m}$ carboxen/polydimethylsiloxane fibers (Supelco, Inc.) were used. The SPME fiber was inserted into the vial and exposed to the gaseous sample for $40 \mathrm{~min}$ at $40^{\circ} \mathrm{C}$. Subsequently, the volatile compounds were de-absorbed in a hot GC injector at $200^{\circ} \mathrm{C}$ for $2 \mathrm{~min}$.

GC/MS analysis. Analysis was performed using a GC/MS (Shimadzu GC-MS QP 2010; Shimadzu,Kyoto,Japan) equipped with a DB-5MS (30 m x $0.250 \mathrm{~mm} \times 0.25 \mu \mathrm{m}$ ) (Agilent Technologies, Inc., Santa Clara, CA, USA) plot column. Injections were conducted in the splitless mode. The temperature of the injector was $200^{\circ} \mathrm{C}$. The flow rate of the helium (99.999\%) carrier gas was maintained constantly at $2 \mathrm{ml} \mathrm{min}^{-1}$. The column temperature was maintained at $40^{\circ} \mathrm{C}$ for 2 min to concentrate the hydrocarbons at the head of the column. Subsequently, the column temperature was increased $7^{\circ} \mathrm{C}$ $\min ^{-1}$ to $200^{\circ} \mathrm{C}$ for $1 \mathrm{~min}$ and following this, was ramped $20^{\circ} \mathrm{C}$ $\mathrm{min}^{-1}$ to $230^{\circ} \mathrm{C}$ for $3 \mathrm{~min}$. The MS analyses were performed in full-scan mode using a scan range of 35-200 amu. The ion source was maintained at $230^{\circ} \mathrm{C}$, and an ionization energy of $70 \mathrm{eV}$ was used for each measurement.

Extraction and pretreatment of the GC/MS raw data. Raw GC/MS data were converted into CDF format (NetCDF) files using Shimadzu GC/MS Postrun Analysis software and processed using the XCMS toolbox (http://metlin.scripps. $\mathrm{edu} / \mathrm{xcms} /$ ).

Statistical analyses. Total area normalization was performed prior to the statistical analysis. Normalized data were subsequently exported to SIMCA-p 11.5 (Umetrics, Malmo, Sweden) for principal component analysis (PCA) and a partial least-squares discriminant analysis (PLSDA) was performed. To guard against overfitting, the default seven-round cross-validation in the SIMCA-p software was applied, and permutation tests with 100 iterations were also carried out to further validate the supervised model. In addition, the two-sided Welch two sample t-test was performed to determine the significance of each metabolite. Based on the variable importance in the projection (VIP values) from the PLSDA model and P-values from t-tests with thresholds of 1.2 and 0.05 , potential metabolic biomarkers were selected. $\mathrm{P}<0.05$ was considered to indicate a statistically significant difference.

\section{Results}

$V O C s$ in RCC. The VOCs in the urine samples of the 22 patients with RCC and 25 healthy subjects were analyzed by GC/MS. Between the patients with RCC and the healthy subjects, a two-dimensional PCA score plot was obtained using 207 parameters, which showed a good separation tendency (Fig. 1A). A PLSDA score plot was subsequently used to separate these two groups with three components $\left(\mathrm{R}^{2} \mathrm{X}=0.763, \mathrm{R}^{2} \mathrm{Y}=0.791\right.$, and $\mathrm{Q}^{2}=0.702$ ) (Fig. 1B). A validation plot obtained from 100 permutation tests showed all of the $\mathrm{R}^{2}$ and $\mathrm{Q}^{2}$ values calculated from the permutated data were lower than the original values (Fig. 1C). Between preoperative and postoperative patients with RCC, we obtained 524 variables and another PCA score plot (Fig. 2A), PLSDA score plot (Fig. 2B) and a validation plot (Fig. $2 \mathrm{C}$ ) were created by repeating the above steps. The results of these plots demonstrated a good PCA separation tendency. The PLSDA separated the patients with two components $\left(R^{2} X=0.614, R^{2} Y=0.879\right.$, and $\left.Q^{2}=0.833\right)$. All of the VIP values of the examined factors in the PLSDA model were calculated. The distinct metabolic biomarkers were selected based on the standard of a VIP value $>1.2$ using the NIST 11 database with a similarity threshold of $75 \%$ (Tables I and II).

\section{Discussion}

Currently, the relatively common VOC samples include breath, blood and urine. Studies have confirmed specific and distinct combinations of VOCs from patients with a variety of cancers (including lung, breast and colorectal cancer) (16-19). Urinary VOC metabolites are more useful biomarkers compared to the VOCs identified in breath and blood for assessing VOC 
A

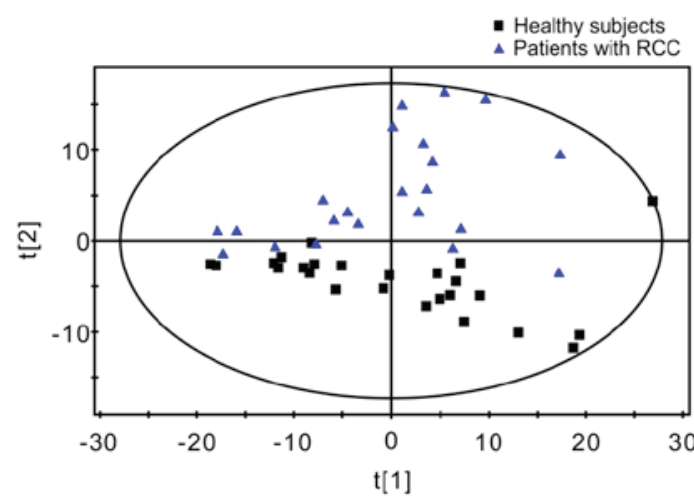

B

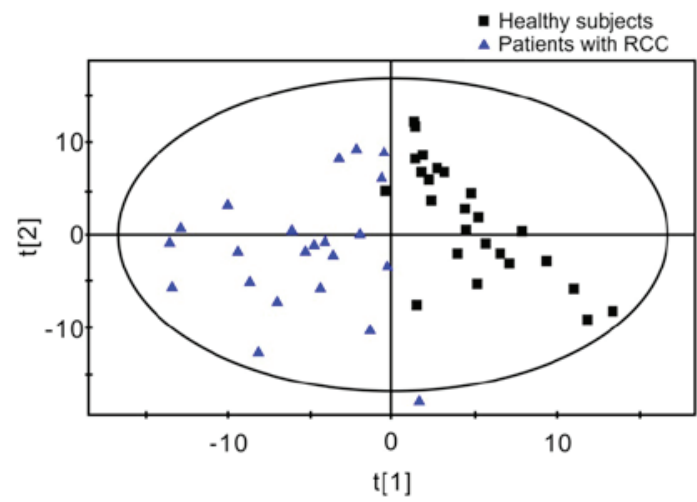

C

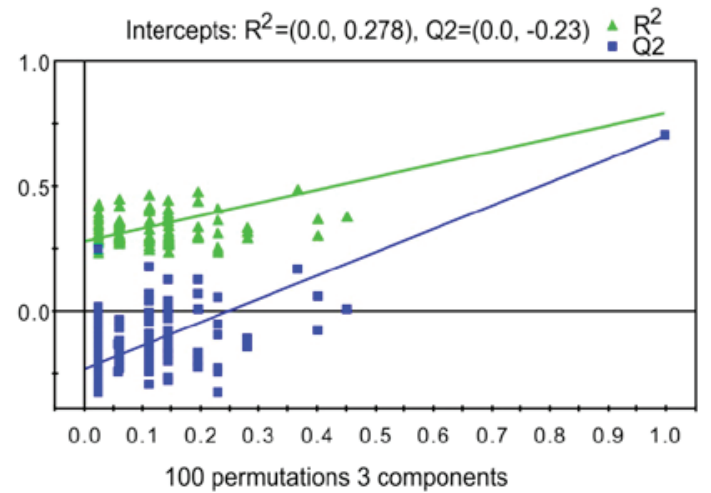

Figure 1. (A) PCA and (B) PLSDA models between the patients with RCC and the healthy subjects $\left(R^{2} X=0.763, R^{2} Y=0.791\right.$, and $\left.Q^{2}=0.702\right)$. (C) Validation plot obtained from 100 permutation tests; Y-intercepts: $\mathrm{R}^{2}=(0.0,0.278)$, $\mathrm{Q}^{2}=(0.0,-0.23)$. PCA, principal component analysis; PLSDA, partial least-squares discriminant analysis; RCC, renal cell carcinoma.

exposure, as urinary metabolites have longer physiological half-lives and sampling is non-invasive (6). The micromolecular metabolites of RCC cells are freely filtered into the urine (15). Urine sampling may avoid the interference of uncertain primary or secondary metabolites compared with breath or blood. The present study acquired the expected VOC profiles from the urine of RCC patients.

Compared to the healthy subjects, patients with RCC showed increased levels of VOC biomarkers, including phenol; decanal; 1,6-dioxacyclododecane-7,12-dione; 1-bromo-1-(3-methyl-1 -pentenylidene)-2,2,3,3-tetramethyl-cyclopropane; nonanal; 3-ethyl-3-methylheptane; isolongifolene-5-ol; 2,5-cyclohexadiene-1,4-dione, 2,6-bis(1,1-dimethylethyl); tetradecane; aniline; and 2,6,10,14-tetramethyl-pentadecane. Three components were decreased, which were styrene; 4-heptanone; and dimethyl silanediol.
A

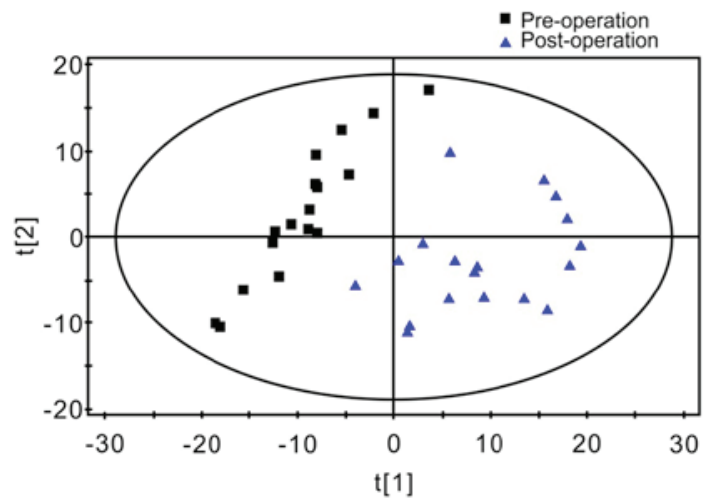

B

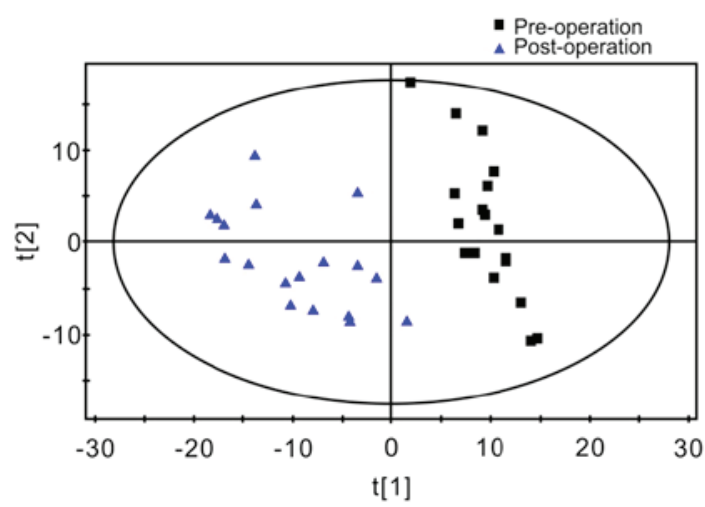

C

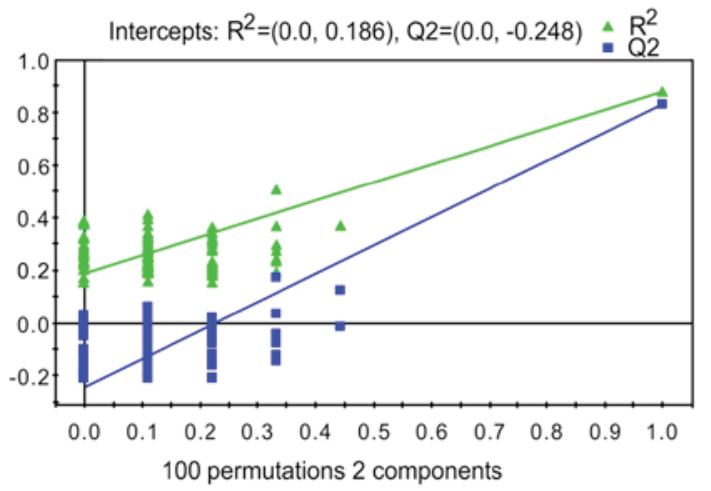

Figure 2. (A) PCA and (B) PLSDA models between preoperative and postoperative patients with $\mathrm{RCC}\left(\mathrm{R}^{2} \mathrm{X}=0.614, \mathrm{R}^{2} \mathrm{Y}=0.879\right.$, and $\left.\mathrm{Q}^{2}=0.833\right)$. (C) Validation plot obtained from 100 permutation tests; Y-Intercepts: $\mathrm{R}^{2}=(0.0$, $0.186), \mathrm{Q}^{2}=(0.0,-0.248)$. PCA, principal component analysis; PLSDA, partial least-squares discriminant analysis; RCC, renal cell carcinoma.

The results showed that 11 biomarkers were increased in RCC patients. These biomarkers were mostly aldehydes, ketones, alkenes, alkanes and substituted benzene compounds, which are produced in response to oxidative stress (20), although the mechanisms by which these compounds were produced remain to be elucidated. 2,6,10,14-Tetramethyl-pentadecane, a strong oxidant, was identified among these compounds, suggesting that oxidative stress may be involved. Decanal is a linear aldehyde that is formed from the oxidation of the majority of the unsaturated fatty acids found in human skin lipids (21). Pentadecane is possibly derived from local oxidative stress in cancer cells, as tumor cells are connected with the lipid peroxidation of cell membranes (22). Additionally, an increase in the aniline levels in the urine of the patients with RCC was observed, which was consistent with the results of Gao et al (22), who found increased aniline levels in the 
Table I. Specific volatile organic compound biomarkers identified at abnormal levels in the urine between patients of RCC and healthy subjects.

\begin{tabular}{|c|c|c|c|c|}
\hline Potential biomarker & $\mathrm{RT}$, min & VIP & P-value & $\mathrm{FC}$ \\
\hline Styrene & 6.069096 & 1.9154 & $1.87 \times 10^{-07}$ & -12.09 \\
\hline Phenol & 8.157637 & 1.9006 & $3.11 \times 10^{-05}$ & 2.7 \\
\hline Decanal & 13.2248 & 1.7131 & $2.14 \times 10^{-05}$ & 3.04 \\
\hline 1,6-Dioxacyclododecane-7,12-dione & 19.72507 & 1.6994 & $1.35 \times 10^{-05}$ & 3.83 \\
\hline $\begin{array}{l}\text { 1-bromo-1-(3-methyl-1-pentenylidene)-2,2,3,3-tetramethyl } \\
\text {-cyclopropane }\end{array}$ & 18.9668 & 1.45 & $5.36 \times 10^{-05}$ & 0.57 \\
\hline Isolongifolene-5-ol & 18.39984 & 1.4163 & $1.21 \times 10^{-03}$ & 1.17 \\
\hline Nonanal & 11.02492 & 1.3884 & $2.67 \times 10^{-03}$ & 2.99 \\
\hline 2,5-Cyclohexadiene-1,4-dione, 2,6-bis(1,1-dimethylethyl)- & 18.3866 & 1.3356 & $1.79 \times 10^{-03}$ & 1.36 \\
\hline 4-Heptanone & 5.650597 & 1.3052 & $8.09 \times 10^{-04}$ & -2.23 \\
\hline Tetradecane & 18.3769 & 1.2991 & $3.65 \times 10^{-03}$ & 1.09 \\
\hline 3-Ethyl-3-methylheptane & 9.890793 & 1.2973 & $2.19 \times 10^{-03}$ & 4.66 \\
\hline Dimethyl-silanediol & 3.061324 & 1.2879 & $1.59 \times 10^{-03}$ & -6.31 \\
\hline Aniline & 8.10944 & 1.2527 & $2.54 \times 10^{-02}$ & 0.69 \\
\hline 2,6,10,14-Tetramethyl-pentadecane & 22.31222 & 1.2163 & $3.79 \times 10^{-03}$ & 1.45 \\
\hline
\end{tabular}

RCC, renal cell carcinoma; RT, retention time; VIP, variable importance in the projection; FC, fold-change: $\mathrm{FC}=\log 10(\mathrm{X} 2 / \mathrm{X} 1)$; X1, arithmetic mean value of a certain metabolite in the case group; X2, arithmetic mean value in the control group. FC with a positive value indicates that the concentration of a certain metabolite is relatively higher in RCC patients compared with the control group.

Table II. Specific volatile organic compound biomarkers identified at abnormal levels in the urine between preoperative and postoperative patients of RCC.

\begin{tabular}{lccr}
\hline Potential biomarker & RT, min & VIP & P-value \\
\hline 2-Ethyl-1-hexanol & 9.260928 & 1.4498 & $4.01 \times 10^{-09}$ \\
6-t-Butyl-2,2,9,9-tetramethyl-3,5-decadien-7-yne & 18.96002 & 1.2408 & $2.70 \times 10^{-07}$ \\
Cyclohexanone & 6.147346 & 1.2383 & $5.12 \times 10^{-06}$ \\
\hline
\end{tabular}

RCC, renal cell carcinoma; RT, retention time; VIP, variable importance in the projection; FC, fold-change: FC= $\log 10(\mathrm{X} 2 / \mathrm{X} 1)$; X1, arithmetic mean value of a certain metabolite in the case group; X2, arithmetic mean value in the control group. FC with a positive value indicates that the concentration of a certain metabolite is relatively higher in RCC patients compared with the control group.

blood of the patients with RCC. The accumulation of another substituted benzene compound, phenol, may accelerate the progress of RCC, as RCC is more commonly found in patients with end-stage renal failure (23).

Humans regulate the balance of water and electrolytes, and eliminate metabolic waste through urine. Ketones were reported to be fairly abundant in human urine (24). Therefore, any impairment of kidney function may significantly affect the excretion of ketones. Urinary 4-heptanone, a type of ketone, was consistently decreased in the patients with RCC. The metabolic pathways of 4-heptanone is known. In hemodialysis patients, di(2-ethylhexyl) phthalate, a plasticizer, is rapidly metabolized to 2-ethylhexanol, which is subsequently oxidized to 2-ethylhexanoic acid and finally to 4-heptanone (25). Halliwell et al (26) reported that 4-heptanone levels are increased in the blood and breath of end-stage renal disease patients. The decrease in 4-heptanone levels found in the urine of the RCC patients suggests that it is difficult for RCC patients to excrete 4-heptanone though the urine; therefore, 4-heptanone remains in other tissues, leading to increased levels in the blood and breath. However, the mechanism of this phenomenon remains to be elucidated. Another decreased biomarker, styrene, is a known carcinogenic environmental toxin. Styrene can attack tissues by leaking into the cytoplasm and causing oxidative damage to proteins (27). Decreased excretion of this carcinogen in the urine can cause accumulation in the kidneys, which leads to an increased risk of cancer.

Between the preoperative and postoperative patients with RCC, three different biomarkers were obtained: 2-Ethyl-1-hexanol, cyclohexanone and 6-t-butyl-2,2,9,9-tetramethyl-3,5-decadien-7-yne. Cyclohexanone is a uremic toxin (9) and levels have been found increased in the blood of patients with end-stage renal disease (26). Cyclohexanone levels were increased in preoperative patients with RCC. Filipiak et al (9) determined that certain compounds, such as butyl acetate and 2-methylpropanal, are consumed when tumor 
cells proliferate. We speculated that 6-t-butyl-2,2,9,9-tetramethyl-3,5-decadien-7-yne could also be overly used by tumor consumption mechanisms. This increased consumption may also account for the decreased levels of 6-t-butyl-2,2,9,9-tetramethyl-3,5-decadien-7-yne in preoperative patients with RCC.

In conclusion, compared with the healthy subjects, RCC has a unique VOC profile, suggesting that this profile may be useful as a diagnostic assay for RCC.

\section{Acknowledgements}

Financial support included grants from the National Natural Science Foundation of China (no. 81402462), China Postdoctoral Science Foundation (no. 2013M531069), Foundation of Heilongjiang Educational Committee (no. 12531245) and the Doctoral Fund of the First Affiliated Hospital of Harbin Medical University (no. 2012B006), which are gratefully acknowledged.

\section{References}

1. Ferlay J, Shin HR, Bray F, Forman D, Mathers C and Parkin DM: Estimates of worldwide burden of cancer in 2008: GLOBOCAN 2008. Int J Cancer 127: 2893-2917, 2010.

2. Rini BI, Campbell SC and Escudier B: Renal cell carcinoma. Lancet 373: 1119-1132, 2009.

3. Guo HF, Song Y and Na YQ: Value of abdominal ultrasound scan, $\mathrm{CT}$ and MRI for diagnosing inferior vena cava tumour thrombus in renal cell carcinoma. Chin Med J (Engl) 122: 2299-2302, 2009

4. Khan AR, Anwar K, Fatima N and Khan SF: Comparison of CT scan and colour flow Doppler ultrasound in detecting venous tumour thrombous in renal cell carcinoma. J Ayub Med Coll Abbottabad 20: 47-50, 2008

5. Rendon RA, Kapoor A, Breau R, Leveridge M, Feifer A, Black PC and So A: Surgical management of renal cell carcinoma: Canadian Kidney Cancer Forum Consensus. Can Urol Assoc J 8: E398-E412, 2014.

6. Altomare DF, Di Lena M, Porcelli F, Trizio L, Travaglio E, Tutino M, Dragonieri S, Memeo V and de Gennaro G: Exhaled volatile organic compounds identify patients with colorectal cancer. Br J Surg 100: 144-150, 2013.

7. Ikeda A, Nishiumi S, Shinohara M, Yoshie T, Hatano N, Okuno T, Bamba T, Fukusaki E, Takenawa T, Azuma T, et al: Serum metabolomics as a novel diagnostic approach for gastrointestinal cancer. Biomed Chromatogr 26: 548-558, 2012.

8. Wu H, Xue R, Dong L, Liu T, Deng C, Zeng H and Shen X: Metabolomic profiling of human urine in hepatocellular carcinoma patients using gas chromatography/mass spectrometry. Anal Chim Acta 648: 98-104, 2009.

9. Filipiak W, Sponring A, Mikoviny T, Ager C, Schubert J, Miekisch W, Amann A and Troppmair J: Release of volatile organic compounds (VOCs) from the lung cancer cell line CALU-1 in vitro. Cancer Cell Int 8: 17, 2008

10. Zambonin CG, Aresta A, Palmisano F, Specchia G and Liso V: Liquid chromatographic determination of urinary 5-methyl-2'-deoxycytidine and pseudouridine as potential biological markers for leukaemia. J Pharm Biomed Anal 21: 1045-1051, 1999.

11. Sasco AJ, Rey F, Reynaud C, Bobin JY, Clavel M and Niveleau A: Breast cancer prognostic significance of some modified urinary nucleosides. Cancer Lett 108: 157-162, 1996.
12. Zheng YF, Yang J, Zhao XJ, Feng B, Kong HW, Chen YJ, Lv S, Zheng MH and Xu GW: Urinary nucleosides as biological markers for patients with colorectal cancer. World J Gastroenterol 11: 3871-3876, 2005

13. Yang J, Xu G, Zheng Y, Kong H, Pang T, Lv S and Yang Q: Diagnosis of liver cancer using HPLC-based metabonomics avoiding false-positive result from hepatitis and hepatocirrhosis diseases. J Chromatogr B Analyt Technol Biomed Life Sci 813: 59-65, 2004.

14. Ganti S and Weiss RH: Urine metabolomics for kidney cancer detection and biomarker discovery. Urol Oncol 29: 551-557, 2011.

15. Jobu K, Sun C, Yoshioka S, Yokota J, Onogawa M, Kawada C, Inoue K, Shuin T, Sendo T and Miyamura M: Metabolomics study on the biochemical profiles of odor elements in urine of human with bladder cancer. Biol Pharm Bull 35: 639-642, 2012.

16. Phillips M, Cataneo RN, Cummin AR, Gagliardi AJ, Gleeson K, Greenberg J, Maxfield RA and Rom WN: Detection of lung cancer with volatile markers in the breath. Chest 123: 2115-2123, 2003.

17. Hietanen E, Bartsch H, Béréziat JC, Camus AM, McClinton S, Eremin O, Davidson L and Boyle P: Diet and oxidative stress in breast, colon and prostate cancer patients: A case-control study. Eur J Clin Nutr 48: 575-586, 1994.

18. Wang C, Li P, Lian A, Sun B, Wang X, Guo L, Chi C, Liu S, Zhao W, Luo S, et al: Blood volatile compounds as biomarkers for colorectal cancer. Cancer Biol Ther 15: 200-206, 2014.

19. Alwis KU, Blount BC, Britt AS, Patel D and Ashley DL: Simultaneous analysis of 28 urinary VOC metabolites using ultra high performance liquid chromatography coupled with electrospray ionization tandem mass spectrometry (UPLC-ESI/MSMS). Anal Chim Acta 750: 152-160, 2012.

20. Nicolaides N: Skin lipids: Their biochemical uniqueness. Science 186: 19-26, 1974.

21. Broza YY, Kremer R, Tisch U, Gevorkyan A, Shiban A, Best LA and Haick H: A nanomaterial-based breath test for short-term follow-up after lung tumor resection. Nanomedicine 9: 15-21, 2013.

22. Gao H, Dong B, Liu X, Xuan H, Huang Y and Lin D: Metabonomic profiling of renal cell carcinoma: High-resolution proton nuclear magnetic resonance spectroscopy of human serum with multivariate data analysis. Anal Chim Acta 624: 269-277, 2008.

23. Mochalski P, Krapf K, Ager C, Wiesenhofer H, Agapiou A, Statheropoulos M, Fuchs D, Ellmerer E, Buszewski B and Amann A: Temporal profiling of human urine VOCs and its potential role under the ruins of collapsed buildings. Toxicol Mech Methods 22: 502-511, 2012.

24. Wahl HG, Hong Q, Hildenbrand S, Risler T, Luft D and Liebich H: 4-Heptanone is a metabolite of the plasticizer di(2-ethylhexyl) phthalate (DEHP) in haemodialysis patients. Nephrol Dial Transplant 19: 2576-2583, 2004.

25. Mochalski P, King J, Haas M, Unterkofler K, Amann A and Mayer G: Blood and breath profiles of volatile organic compounds in patients with end-stage renal disease. BMC Nephrol 15: 43, 2014.

26. Halliwell B, Gutteridge JM and Cross CE: Free radicals, antioxidants, and human disease: Where are we now? J Lab Clin Med 119: 598-620, 1992.

27. Thompson-Torgerson CS, Champion HC, Santhanam L, Harris ZL and Shoukas AA: Cyclohexanone contamination from extracorporeal circuits impairs cardiovascular function. Am J Physiol Heart Circ Physiol 296: H1926-H1932, 2009. 